\title{
Marek's disease vaccines-induced differential expression of known and novel microRNAs in primary lymphoid organ bursae of White Leghorn
}

Lei Zhang ${ }^{1,2 \dagger}$, Chen Zhu ${ }^{1,3 \dagger}$, Mohammad Heidari ${ }^{1}$, Kunzhe Dong ${ }^{1,4}$, Shuang Chang ${ }^{1,5}$, Qingmei Xie ${ }^{1,6}$ and Huanmin Zhang ${ }^{1 *}$ (1)

\begin{abstract}
Marek's disease (MD) is a contagious disease of domestic chickens caused by MD viruses. MD has been controlled primarily by vaccinations, yet sporadic outbreaks of MD take place worldwide. Commonly used MD vaccines include HVT, SB-1 and CVI988/Rispens and their efficacies are reportedly dependent of multiple factors including host genetics. Our previous studies showed protective efficacy of a MD vaccine can differ drastically from one chicken line to the next. Advanced understanding on the underlying genetic and epigenetic factors that modulate vaccine efficacy would greatly improve the strategy in design and development of more potent vaccines. Two highly inbred lines of White Leghorn were inoculated with HVT and CVI988/Rispens. Bursa samples were taken 26 days post-vaccination and subjected to small RNA sequencing analysis to profile microRNAs (miRNA). A total of 589 and 519 miRNAs was identified in one line, known as line $\sigma_{3}, 490$ and 630 miRNAs were identified in the other, known as line $7_{2}$, in response to HVT or CVI988/Rispens inoculation, respectively. HVT and CVI988/Rispens induced mutually exclusive 4 and 13 differentially expressed (DE) miRNAs in line $\sigma_{3}$ birds in contrast to a non-vaccinated group of the same line. HVT failed to induce any DE miRNA and CVI988/Rispens induced a single DE miRNA in line $7_{2}$ birds. Thousands of target genes for the DE miRNAs were predicted, which were enriched in a variety of gene ontology terms and pathways. This finding suggests the epigenetic factor, microRNA, is highly likely involved in modulating vaccine protective efficacy in chicken.
\end{abstract}

\section{Introduction}

Marek's disease (MD) is a proliferative disease of domestic chickens caused by Gallid alphaherpesvirus 2 (Herpesviridae), commonly known as MD virus (MDV). MD was first described by József Marek, a Hungarian veterinarian [1]. Clinical symptoms of MD vary among different lines of chickens and are dependent of exposure to

\footnotetext{
*Correspondence: Huanmin.Zhang@usda.gov

'Lei Zhang and Chen Zhu contributed equally to this work

${ }^{1}$ Avian Disease and Oncology Laboratory, USDA-ARS, East Lansing, MI 48823, USA

Full list of author information is available at the end of the article
}

different strains of MDV [2, 3], but commonly include polyneuritis, visceral lymphoma, acute transient paralysis, immunosuppression, brain edema, and acute rash [4, 5]. The morbidity and mortality could range from 11 [6] up to $100 \%$ [3]. Although MD has been well under control most of the time in most regions of the world by wide use of MD vaccines and improved management measures since the 1970s [7-9], sporadic outbreaks still occur from time to time all over the world. MD continues to pose a real threat and reportedly costs more than 2 billion U.S. dollars annually to the poultry industry $[9,10]$.

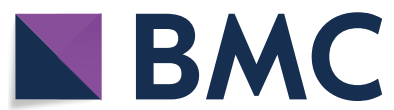

(c) The Author(s) 2020. This article is licensed under a Creative Commons Attribution 4.0 International License, which permits use, sharing, adaptation, distribution and reproduction in any medium or format, as long as you give appropriate credit to the original author(s) and the source, provide a link to the Creative Commons licence, and indicate if changes were made. The images or other third party material in this article are included in the article's Creative Commons licence, unless indicated otherwise in a credit line to the material. If material is not included in the article's Creative Commons licence and your intended use is not permitted by statutory regulation or exceeds the permitted use, you will need to obtain permission directly from the copyright holder. To view a copy of this licence, visit http://creativeco mmons.org/licenses/by/4.0/. The Creative Commons Public Domain Dedication waiver (http://creativecommons.org/publicdomain/ zero/1.0/) applies to the data made available in this article, unless otherwise stated in a credit line to the data. 
There are three commonly used MD vaccines commercially available up to date in most parts of the world. They are CVI988/Rispens, SB-1, and HVT, which were derived from MDV-1, MDV-2, and MDV-3 (also known as serotype 1,2 , and $3 \mathrm{MDV}$ ), respectively [11-13]. The SB-1 and HVT are naturally non-oncogenic. The CVI988/ Rispens is a product of serial passaging in tissue culture and is considered an attenuated strain of live MDV-1. Like SB-1 and HVT, CVI988/Rispens is infectious but incapable of inducing lymphoid organ atrophy or tumor formation [14]. CVI988/Rispens and HVT have been used individually or in different combination of bivalent or trivalent format along with SB-1 on commercial farms worldwide [14]. Most users and researchers believe CVI988/Rispens is the most protective one of all commercially available vaccines against MD in chickens [15, 16], and HVT is no longer sufficiently protective or far less protective than CVI988/Rispens in preventing tumor formation against very virulent strains of MDV induction in chickens [17-20].

Protective efficacy of a vaccine is not only determined by the potency of the vaccine itself, but also host genetics of the recipients. By using $B$-congenic lines of experimental chickens [21] in trials of vaccination followed by MDV challenge, it was clearly shown that chicken major histocompatibility complex $(\mathrm{MHC}) \mathrm{B}$-haplotype poses significant influence on vaccinal immunity against MD [22]. This important finding was also subsequently demonstrated to hold true in commercial chickens [23]. Based on the data from those studies, chickens of $B^{*} 2 / B^{*} 2$, $B * 13 / B * 13, B * 15 / B * 15$ and $B * 21 / B * 21$ haplotypes gain the best protection against MD by MDV-1 vaccines like CVI988/Rispens; $B * 5 / B * 5$ haplotype chickens are better protected by MDV-2 vaccines like SB-1; and in those genetic lines, $B * 2 / B * 2$ and $B * 13 / B * 13$ chickens were not better protected against MD by none of the MDV-1, MDV-2, and MDV-3 vaccines in contrast to other haplotypes of chickens.

In addition to $B$-haplotypes, non- $M H C$ host genetic components also play a significant role modulating $\mathrm{MD}$ vaccine efficacy against MD [24]. The two highly inbred genetic lines of chickens, lines $6_{3}$ and $7_{2}$, maintained at USDA, Agricultural Research Service, Avian Disease and Oncology Laboratory (East Lansing, Michigan, USA), share a common $B * 2$ haplotype, but line $6_{3}$ is relatively resistant and line $7_{2}$ is highly susceptible to MD [3]. Data from vaccination and challenge trials conducted in recent years showed that line $6_{3}$ chickens can convey better or equivalent protective efficacy in response to MDV-3 HVT vaccination than MDV-1 CVI988/Rispens. In a sharp contrast, the line $7_{2}$ birds respond to HVT very poorly and can convey some protection in response to CVI988/Rispens [25].
As early as in the 1980s, researchers realized that new routes in development of safer and more effective vaccines could be opened upon advances in molecular biology, genetics, and epigenetics [26, 27]. New evidences in the molecular, genomic and epigenomic levels in response to vaccination should highly likely lead to better understanding on protective efficacy of vaccines against infectious diseases including virus-induced cancers and offer new insights of host immune defenses as well as interaction between host immune system and vaccines. This study was designed to profile microRNAs (miRNA) in two genetically divergent lines of chicken post MD vaccine inoculation and to explore differentially expressed miRNAs in response to vaccination by CVI988/Rispens or HVT in contrast to non-vaccinated birds.

\section{Materials and methods}

\section{Experimental animals}

Day-old chicks were randomly sampled from two highly inbred lines of White Leghorns, known as the line $6_{3}$ and line $7_{2}$ chickens, maintained on the Avian Disease and Oncology Laboratory farm at East Lansing, Michigan, USA. Both lines are $B * 2$ haplotype homozygous but drastically differ in genetic resistance to MD. Line $6_{3}$ is resistant and line $7_{2}$ is highly susceptible [21]. The two lines of chickens also drastically differ in response to MD vaccines [28].

\section{Challenge trial}

The sampled 1-day old chicks from line $6_{3}$ and line $7_{2}$ were randomly divided into two groups per line. One group was inoculated with HVT intraperitoneally (IP) at a dose of 2000 plaque-forming units (PFU) each; and the other group was inoculated with CVI988/Rispens (IP) with the same dose. In addition, a control group was also included with the same sample size for each of the chicken lines under the same conditions in conjunction with a joint project simultaneously (to minimize the use of animals per experiment). The corresponding control group datasets were used in this study only to examine the differential expression of miRNAs in response to HVT and CVI988/Rispens inoculation. All chickens used in this study were housed in a BSL-2 experimental facility during the trial. Feed and water were supplied ad libitum. The chickens were observed daily throughout the entire duration of the experiment. This animal experiment was approved by USDA, Avian Disease and Oncology Laboratory Institutional Animal Care and Use Committee (IACUC). The IACUC guidelines established and approved by the ADOL IACUC (April 2005) and the Guide for the care and use of Laboratory Animals by Institute for Laboratory Animal Research (2011) were closely followed throughout the experiment. 


\section{Total RNA extraction}

Three chickens from each group were randomly euthanized at 26 days post-inoculation. Bursa samples were individually collected, and immediately placed into RNAlater solution (Qiagen, Valencia, CA, USA). The collected samples were stored at $-20{ }^{\circ} \mathrm{C}$ until extractions of the total RNA samples. Total RNA samples were extracted with TRIzol reagent (Invitrogen, Carlsbad, CA, USA) following the manufacturer's instructions.

\section{Small RNA sequencing}

Total RNA samples were quantitatively and qualitatively checked with a NanoDrop 8000 Spectrophotometer (Thermo Fisher Scientific, Waltham, MA, USA) and an Agilent 2100 Bioanalyzer (Agilent Technologies, Santa Clara, CA, USA), respectively. Good quality RNA samples were chosen to construct standard cDNA libraries using Illumina TruSeq Small RNA Library Preparation kits following the manufacturer's recommendations. Completed libraries were subjected to routine quality control (QC) checks and quantified using a combination of Qubit dsDNA HS and Agilent Bioanalyzer High Sensitivity DNA assays. The libraries were sequenced on an Illumina HiSeq 4000 sequencer using SBS (Sequencing by Synthesis) reagents. Base calling was accomplished by use of Illuima Real Time Analysis (RTA) v2.7.7 and the output of RTA was demultiplexed and then converted to FastQ format data with Ilunima Bcl2fastq v2.19.1 (Illumina, San Diego, CA, USA). The small RNA sequencing datasets supporting the results and conclusions of this article are available in the NCBI SRA repository [29]. The sequence datasets (accession numbers: SAMN11674924 to SAMN11674929) of the unvaccinated line $6_{3}$ and $7_{2}$ control groups used in the comparison analyses of miRNA differential expression were also deposited to NCBI SRA repository. The small RNA sequencing operations, including library preparation and preliminary reads quality control, were performed at the Research Technology Support Facility, Michigan State University (East Lansing, MI, USA).

\section{Small RNA_Seq reads data analyses}

Small RNA_Seq reads data files that passed QC were analyzed one at a time with the miRDeep* software v3.8 [30] using the default parameters except the adapter sequence and the chicken genome build index files. The adapter sequence used in the analysis is TGG AAT TCT CGG GTG CCA AGG AAC TCC AGT CAC (Illumina); and the chicken genome build index (build_bwt_idx) files were constructed based on the chromosome information of the galGal 5.0 genome build. In addition, the "knownMiR.gff" file used in miRDeep* analysis of this study was the "gga.gff3" file at the miRbase download website [31, 32], which was constructed in accordance to galGal 5.0 assembly. Target genes of differentially expressed miRNAs were predicted using the built-in target gene prediction function in miRDeep*, which employees the most commonly used target gene prediction tool, TargetScan, in predicting the target genes of known and novel miRNAs [30, 33].

\section{Droplet digital ${ }^{\mathrm{TM}} \mathrm{PCR}$ analysis}

To validate the miRNA expressions derived from small RNA reads data, identified miRNAs were randomly elected to subject to droplet digital PCR (ddPCR) analysis to collect the absolute quantification reads of each miRNA from each of the total RNA samples of the four treatment groups (line $6_{3} \mathrm{HVT}$, line $6_{3}$ CVI988/Rispens, line $7_{2}$ HVT, and line $7_{2}$ CVI988/Rispens). Primers were designed for each of the selected miRNAs based on its mature sequence as described by Balcells et al. [34], which were used in the ddPCR (QX200 ${ }^{\mathrm{TM}}$ ddPCR system; Bio-Rad Laboratories, Inc., Hercules, CA, USA) analysis. The cDNA samples used in ddPCR validation were reversely transcribed from individual RNA samples using the iScript ${ }^{\mathrm{TM}}$ RT Supermix Kit (Cat No. 170-8841) and following the manufacturer's instructions (Bio-Rad). A ddPCR reaction of $25 \mu \mathrm{L}$ in final volume was initially prepared per miRNA per biological sample containing $2 \mu \mathrm{L}$ of cDNA, $12.5 \mu \mathrm{L}$ of EvaGreen Supermix (Cat No. 1864034), $0.5 \mu \mathrm{L}$ of each forward and reverse primers (200 nM; synthesized by Eurofins Genomics, Huntsville, $\mathrm{AL}$ ), and $9.5 \mu \mathrm{L}$ of nuclease-free water. Of which, $20 \mu \mathrm{L}$ were loaded into one of 8 sample channels of a $\mathrm{DG}^{\mathrm{TM}}$ cartridge (Cat No. 1864008, Bio-Rad). Each oil well was loaded with $70 \mu \mathrm{L}$ of droplet generating oil (Cat No. 1864006, Bio-Rad). The loaded $\mathrm{DG}^{\mathrm{TM}}$ cartridges were placed on a QX200 ${ }^{\mathrm{TM}}$ droplet generator (Bio-Rad) to generate the digital droplets. Forty $\mu \mathrm{L}$ of the generated droplet emulsion for each sample were transferred to a well in a 96-well PCR plate followed by polymerase chain reaction with EvaGreen on a $\mathrm{C} 1000^{\mathrm{TM}}$ Thermal Cycler (Bio-Rad). The cycling conditions were $95{ }^{\circ} \mathrm{C}$ for $5 \mathrm{~min}$, followed by 40 cycles of $95^{\circ} \mathrm{C}$ for $15 \mathrm{~s}, 58^{\circ} \mathrm{C}$ for $60 \mathrm{~s}$, and a final extension step of $98{ }^{\circ} \mathrm{C}$ for $10 \mathrm{~min}$. The droplets post PCR were read well by well on a $\mathrm{QX} 200^{\mathrm{TM}}$ droplet reader (Bio-Rad). PCR-positive and PCR-negative droplets in each of the wells were counted and analyzed with the QuantaSoft ${ }^{\mathrm{TM}}$ Software (Version 1.7, Bio-Rad).

\section{Differentially expressed miRNA identification and GO terms enrichment analysis}

The number of reads per miRNA for each biological sample were counted using HTSeq [35]. In each of the pairwise comparisons (between MD vaccine inoculated 
group and the control group for each chicken line, between the two MD vaccinated groups for each chicken line, and between the two chicken lines for each vaccinated group), differentially expressed miRNAs were identified by use of a custom $\mathrm{R}$ script encompassing the DESeq R package (2.1.0). A filter criterion of $F D R<0.05$ and $\mathrm{FC}>2$ was enforced. For some of the differentially expressed miRNAs that ended up with a zero-statistic estimate for a normalized average TPM (baseMeanA or baseMeanB) in a contrast, an arbitrary small value of 1 was assigned to substitute the zero in order to computer a numeric fold change, and then a $\log _{2}$ fold change value for easier comprehension of the estimates. To better understand the functional involvements of the identified miRNAs differentially expressed in response to the vaccination, predicted target gene lists of differentially expressed miRNAs for each of the contrasts were subjected to GO terms and pathway analysis using the g:Proflier [36] online tools with the following options: Organism: Gallus gallus; Statistical domain scope: All known genes; Significance threshold: Bonferroni correction; User threshold: 0.01 [37].

\section{Results}

\section{Small RNA sequencing}

Small RNA sequencing generated an average of 35.7 million PF reads (the number of clusters that passed Illumina's "Chastity filter") per biological sample, with a range of 23 to 50.8 million PF reads for all 12 bursal samples of the lines $6_{3}$ and $7_{2}$ chickens inoculated with either HVT or CVI988/Rispens. The raw sequence datasets (accession numbers: SAMN11675491 - SMAN11675502) are available at the Sequence Read Archive (SRA) under the National Centre for Biotechnology Information (NCBI) website with an assigned BioProject SRA accession number: PRJNA543526 [38].

\section{MicroRNA profiles}

A total of 693 miRNAs was identified in this study, which were mapped to 31 pairs of chromosomes (gga chr1-28, chr32-33, chrW and Z), and was identified in no fewer than three biosamples with reads counts of five and above. Four hundred and ninety-five out of the 693 miRNAs were novel miRNAs (see Additional file 1 for a complete list of the identified miRNAs), which have been deposited to miRbase Registry for processing in validation and finalizing name assignment. The total numbers of identified miRNAs were 589 and 519 in bursae of the line $6_{3}$ birds, and 490 and 630 in bursae of the line $7_{2}$ birds inoculated with HVT or CVI988/Rispens, respectively (Table 1 ). The numbers of exclusively identified miRNAs and the numbers of identified miRNAs in common between vaccine treatment groups of the line $\sigma_{3}$ and
Table 1 Numbers of microRNAs identified in bursae of line $6_{3}$ and $7_{2}$ chickens 26-days post HVT or CVI988/Rispens inoculation after hatch

\begin{tabular}{lllll}
\hline Chicken & Vaccine & $\begin{array}{l}\text { Total } \\
\text { number }\end{array}$ & $\begin{array}{l}\text { Known } \\
\text { miRNA }\end{array}$ & Novel miRNA \\
\hline Line $6_{3}$ & HVT & 589 & 195 & 394 \\
& CVI988/Risp- & 519 & 187 & 332 \\
& ens & & & \\
Line 72 & HVT & 490 & 188 & 302 \\
& CVI988/Risp- & 630 & 192 & 438 \\
& ens & & & \\
\hline
\end{tabular}

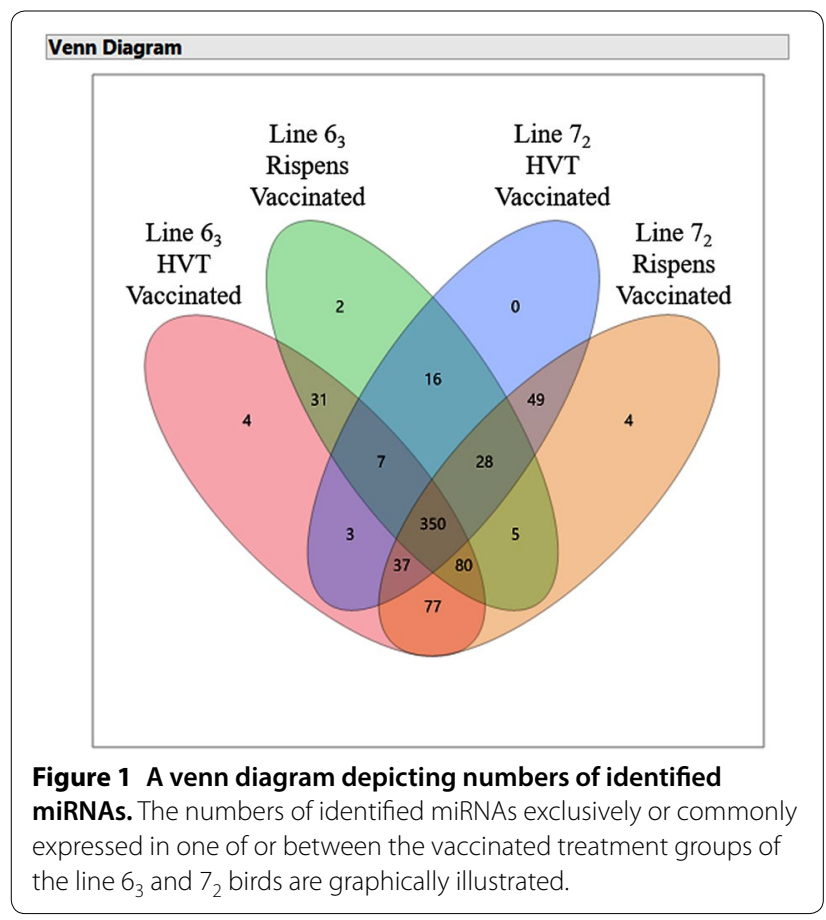

$7_{2}$ birds are depicted in a Venn diagram (Figure 1). The details of the identified miRNAs, including miRNA name (miRNA_ID), average normalized transcripts per million (Mean (TPM)), mature loci, chromosome (chr), hairpin and mature sequences, are given in Additional files 2, 3, 4,5 .

\section{Differentially expressed microRNAs in response to HVT vaccination}

Four out of the 589 miRNAs identified in bursae of the line $6_{3}$ birds were differentially expressed in response to HVT vaccination in contrast to an unvaccinated group of line $6_{3}$ birds $(1.33 \mathrm{E}-6<p<2.58 \mathrm{E}-4$, $9.62 \mathrm{E}-4<F D R<4.68 \mathrm{E}-2)$. Two of the miRNAs were significantly upregulated ( $\log _{2}$ Fold Change: 3.27 and 7.63), 
and the other two were significantly downregulated $\left(\log _{2}\right.$ FC: -8.68 and -6.09$)$. None of the identified miRNAs in bursae of the line $7_{2}$ birds was differentially expressed in response to HVT vaccination in contrast to its counterpart of an unvaccinated group (Table 2).

\section{Differentially expressed microRNAs in response to CVI988/ Rispens vaccination}

One of the novel miRNAs, novelMiR_215, was significantly downregulated $(p=2.14 \mathrm{E}-10, F D R=1.72 \mathrm{E}-08)$ with a $\log _{2} \mathrm{FC}=-4.69$, and 12 miRNAs were significantly upregulated $1.60 \mathrm{E}-134<p<7.65 \mathrm{E}-4$, $1.16 \mathrm{E}-131<F D R<4.27 \mathrm{E}-2)$ with a range of $\log _{2} \mathrm{FC}$ from 3.06 to 10.77 in bursae of line $6_{3}$ birds in response to CVI988/Rispens vaccination. In bursae of line $7_{2}$ birds, CVI988/Rispens induced a single novel miRNA, novelMiR_1251, with significantly downregulated expression $\left(\log _{2} \mathrm{FC}=-10.01, p<4.53 \mathrm{E}-5, F D R=3.43 \mathrm{E}-2\right)$ in contrast to line $7_{2}$ unvaccinated group (Table 2 ).

Differentially expressed microRNAs in response to CV1988/ Rispens in contrast to HVT vaccination within each of the chicken lines

Twenty-eight identified miRNAs were differentially expressed in bursae of the line $6_{3}$ birds in response to CVI988/Rispens inoculation in contrast to HVT inoculation. Four of the miRNAs were significantly downregulated with $\log _{2} \mathrm{FC}$ ranged from -7.67 to -3.75 $(4.76 \mathrm{E}-13<p<1.32 \mathrm{E}-5 ; \quad 3.25 \mathrm{E}-11<F D R<5.22 \mathrm{E}-4)$. The other 24 miRNAs were significantly upregulated. The $\log _{2}$ FC ranged from 1.51 up to 11.26 $(3.05 \mathrm{E}-192<p<1.73 \mathrm{E}-3 ; \quad 2.29 \mathrm{E}-189<F D R<4.49 \mathrm{E}-2)$. In contrast to HVT inoculation, CVI988/Rispensinduced a single novel microRNA, novelMiR_1251, with significantly downregulated expression $\left(\log _{2}\right.$ $\mathrm{FC}=-11.45 ; \quad p<1.80 \mathrm{E}-7 \quad$ and $F D R<1.37 \mathrm{E}-4)$ and another novel microRNA, novelMiR_215, with significantly upregulated expression $\left(\log _{2} \quad \mathrm{FC}=3.21\right.$; $p<1.70 \mathrm{E}-4$ and $F D R<4.29 \mathrm{E}-2$ ) in line 72 birds (Table 2). The numbers of differentially expressed miRNAs in response to HVT or CVI988/Rispens inoculation and between the CVI988/Rispens and HVT vaccination within each of the two chicken lines are graphically illustrated in a Venn diagram (Figure 2).

\section{Differentially expressed microRNAs in response to HVT or CVI988/Rispens vaccination between line $\sigma_{3}$ and line $7_{2}$ birds}

There were 34 identified miRNAs ( 8 known-miRNAs and 26 novel miRNAs) differentially expressed in bursae between the line $6_{3}$ and $7_{2}$ birds 26 days post HVT inoculation. Eight of those miRNAs were significantly upregulated ( $\log _{2}$ FC: 3.49 to $7.64 ; 3.66 \mathrm{E}-6<p<5.57 \mathrm{E}-4$;
$1.37 \mathrm{E}-4<F D R<1.31 \mathrm{E}-2)$ and 26 were downregulated $\left(\log _{2}\right.$ FC: -11.45 to $-1.24 ; 6.58 \mathrm{E}-47<p<1.89 \mathrm{E}-3$; $5.09 \mathrm{E}-44<F D R<4.06 \mathrm{E}-2$ ) in line $6_{3}$ birds in contrast to line $7_{2}$ in response to HVT inoculation. Thirty identified miRNAs (6 known-miRNAs and 24 novel miRNAs) were differentially expressed between line $6_{3}$ and 72 birds post CVI988/Rispens inoculation. Twenty-four were significantly upregulated ( $\log _{2} \mathrm{FC}: 1.45$ to 11.29 ; $5.67 \mathrm{E}-101<p<1.97 \mathrm{E}-3 ; \quad 4.43 \mathrm{E}-98<F D R<4.80 \mathrm{E}-2)$, and the other 6 were downregulated $\left(\log _{2}\right.$ FC: $\quad-6.71$ to $-1.69 ; \quad 4.31 \mathrm{E}-21<p<1.59 \mathrm{E}-3$; $1.12 \mathrm{E}-18<F D R<4.01 \mathrm{E}-2)$ in response to CVI988/ Rispens inoculation in line $6_{3}$ birds in contrast to line $7_{2}$ (Table 3).

\section{ddPCR validation of miRNA expression determined by small RNA_Seq}

To validate the miRNA expression determined by small RNA_Seq analysis, ten of the identified miRNAs were selected to subject to absolute quantification of the expression by ddPCR. The selected miRNAs and the designed ddPCR primers are listed in Table 4. The summary statistics including correlation coefficients between the normalized small RNA_Seq reads and the ddPCR absolute quantification counts are given in Table 5. The correlation coefficients ranged from $\mathrm{r}=0.63$ (gga-miR-140) up to $\mathrm{r}=0.99$ (both gga-miR-31 and gga-miR-499) with a single $p$ value smaller than 0.0001 (Table 5). Manhattan bivariate plots depicting the relationship between normalized expression of small RNA_Seq data (TPM) and the ddPCR absolute quantification reads for four of the selected miRNAs are given in Figure 3, which visually illustrates the validation. Taking all together, Table 5 and Figure 4 provided experimental evidence that highly positively supports the microRNA expression estimates derived from the small RNA_Seq data of this study.

Predicted target genes of differentially expressed miRNAs Varied numbers (12 up to 6153) of target genes were predicted for the differentially expressed miRNAs per contrast for all contrast groups except one, the line $7_{2} \mathrm{HVT}$ treatment group over its counterpart of control group, which resulted in no differentially expressed miRNA. The numbers of differentially expressed miRNAs, predicted target genes, involved gene ontology (GO) terms and KEGG pathways are given in Table 6 by contrast group.

Target-gene-set enrichment in GO terms and pathways for differentially expressed microRNAs in response to HVT or CVI988/Riepsnes vaccination of line $\sigma_{3}$ and $7_{2}$ birds

Target genes of differentially expressed miRNAs in response to HVT or CVI988/Rispens in line $6_{3}$ birds 


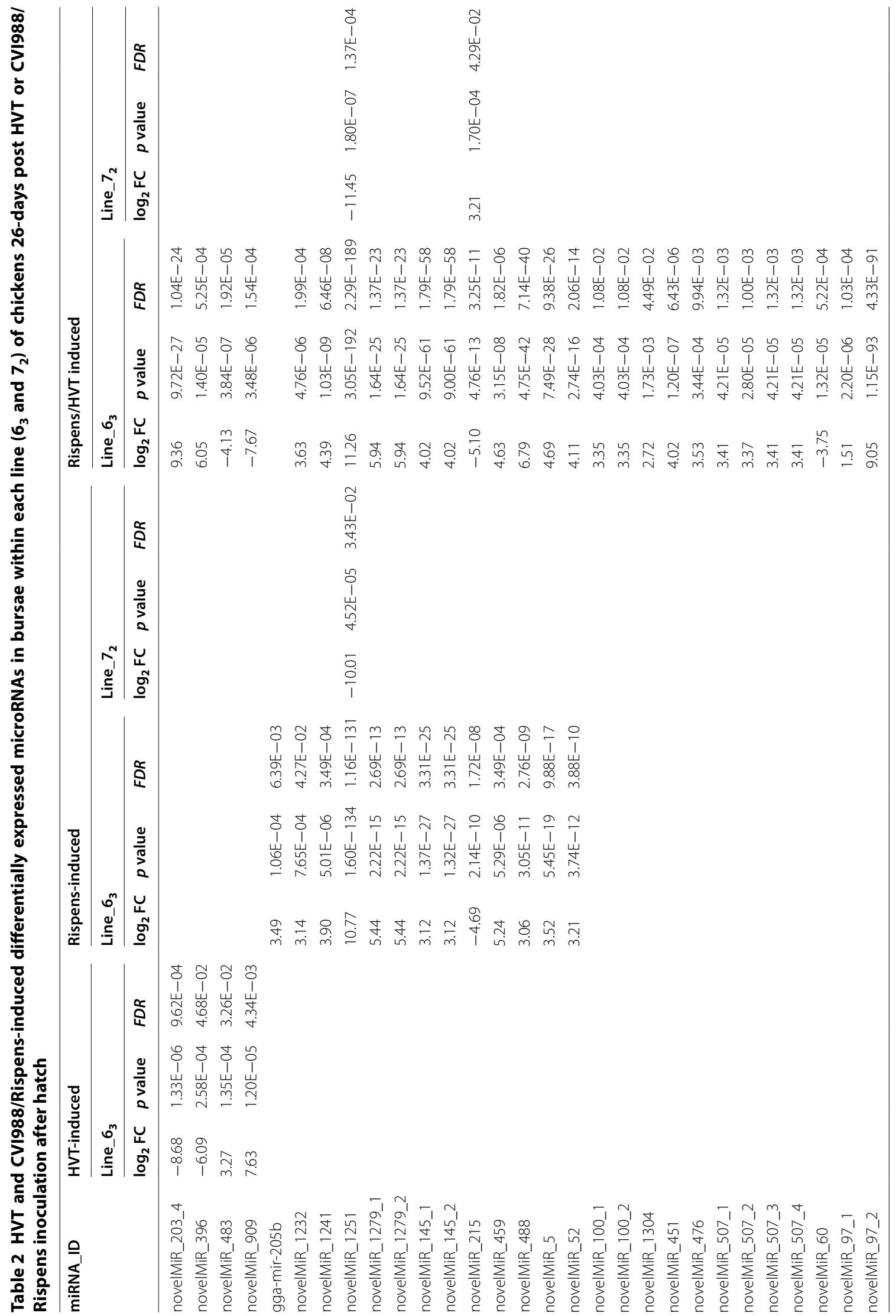




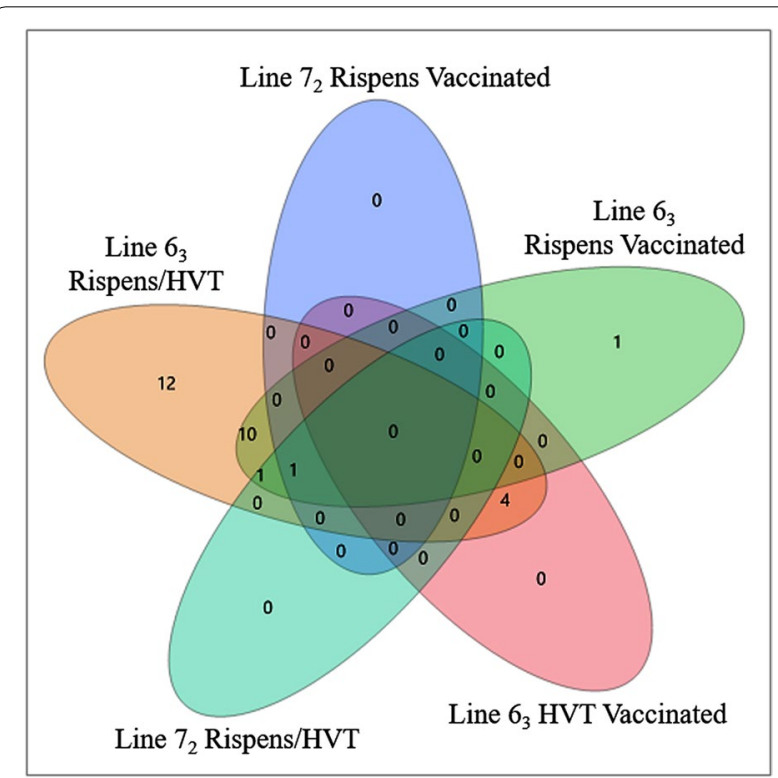

Figure 2 A venn diagram graphically illustrating the numbers of differentially expressed miRNAs. The numbers of differentially expressed miRNAs exclusively in one or commonly between vaccine treatment groups of a chicken line and between the lines as well as the comparisons between vaccine treatments within each of the lines are depicted.

were highly enriched in a variety of GO terms and pathways. In contrast, HVT failed to induce any differentially expressed miRNA and CVI988/Rispens induced a single differentially expressed miRNA in line $7_{2}$ birds, which reportedly targets 12 functional genes. Therefore, there was only a very limited number of GO terms and pathways in which those target genes were enriched.

The target genes of HVT-induced differentially expressed miRNAs in the line $6_{3}$ birds were significantly enriched (Bonferroni corrected $p<0.01$ ) in a total of 237 GO terms, which included 17 molecular function (GO:MF), 172 biological process (GO:BP), and 48 cellular component (GO:CC) terms (Additional file 6; Figure 4), in addition to reactomes (REAC), WiKiPathways (WP), and transcription factors (TF). Most of the MF terms present binding functions, including protein binding, nucleic acid binding, organic cyclic compound binding, heterocyclic compound binding, DNA binding, RNA binding, enzyme binding, ion binding, and sequence-specific DNA binding. The BP terms encompassed cellular process, regulation of cellular and biological processes, regulation of gene expression, cell differentiation, cellular response to stimulus, signaling, signal transduction, and regulation of signal transduction. The CC terms are involved in membrane functionalities including membrane-bounded organelle, intracellular membrane-bounded organelle, membrane-enclosed lumen, membrane part, plasma membrane, intrinsic component of membrane, integral component of membrane, and plasma membrane bounded cell projection.

The target genes of CVI988/Rispens-induced differentially expressed miRNAs in the line $6_{3}$ birds were significantly over-enriched (Bonferroni corrected $p<0.01$ ) in a total of $1057 \mathrm{GO}$ terms, which included $118 \mathrm{GO}$ :MF, 811 GO:BP, and 128 GO:CC terms (Figure 4). MF terms are heavily presented in binding functions including protein binding, ion binding, organic cyclic and heterocyclic compound binding, enzyme binding, anion binding, DNA binding, transcription regulatory region DNA binding, cation binding, small molecule binding, kinase binding, and ATP binding. MF terms are also broadly involved in biological activities including phosphoric ester hydrolase activity, transcription coregulator activity, channel activity, phosphatase activity, protein tyrosine phosphatase activity, ubiquitin protein ligase activity, enzyme activator activity, molecular transducer activity, and signaling receptor activity. BP terms are presented in cell adhesion, communication, cell cycle, cycle phase transition, cycle process, cell death, development, growth, and cell junction assembly and organization, cell migration and motility, cell-cell adhesion, signaling, and signaling by Wnt. The CC terms are involved in centrosome, chromatin, cytoplasm, dendritic tree, endomembrane system, intracellular organelle, organelle lumen, and intracellular vesicle. KEGG pathways including MAPK signaling pathway, Wnt signaling pathway, Hedgehog signaling pathway, and mTOR signaling pathway (Additional file 7).

Target genes of differentially expressed miRNAs between line $6_{3}$ and line $7_{2}$ birds in response to HVT vaccination were also heavily enriched in (221) MF terms, (1528) BP terms, and (250) CC terms. MF terms included variety of binding functions, such as ankyrin binding, ATP binding, ATPase binding, GTP binding, cytokine receptor binding, mRNA binding, myosin binding, and nuclear hormone receptor binding. MF terms also cover pyrophosphatase activity, Ras guanylnucleotide exchange factor activity, signaling activity, and signaling receptor activity. The BP terms involved in a variety of systems and functions including angiogenesis, apoptotic process, autophagy, biological adhesion, regulation and process, cell differentiation and division, cell-substrate adhesion and junction assembly, and cellular response to varied compounds and stimulus. The CC terms are involved in functions including cell surface, coated membrane, vesicle, vesical membrane, transcription factor complex, and whole membrane. In addition, the target genes were also enriched in KEGG pathways including adipocytokine signaling pathway, calcium signaling pathway, C-type lectin 
Table 3 Differentially expressed microRNAs in bursae between the line $\mathbf{6}_{\mathbf{3}}$ and the line $\mathbf{7}_{\mathbf{2}}$ birds 26 -days post HVT or CV1988/Rispens inoculation after hatch

\begin{tabular}{|c|c|c|c|c|c|c|}
\hline \multirow[t]{3}{*}{ miRNA_ID } & \multicolumn{6}{|c|}{ Line $-\sigma_{3} /$ Line ${ }_{-} 7_{2}$} \\
\hline & \multicolumn{3}{|c|}{ HVT-induced } & \multicolumn{3}{|c|}{ Rispens-induced } \\
\hline & $\log _{2} F C$ & $p$ value & $F D R$ & $\log _{2} F C$ & $p$ value & $F D R$ \\
\hline gga-mir-1684a & -4.99 & $4.21 \mathrm{E}-13$ & $1.09 \mathrm{E}-10$ & -6.71 & $8.25 E-10$ & $7.16 \mathrm{E}-08$ \\
\hline gga-mir-193b & -1.95 & $9.35 \mathrm{E}-06$ & 4.30E-04 & -1.69 & $1.27 \mathrm{E}-03$ & $3.29 \mathrm{E}-02$ \\
\hline gga-mir-31 & -1.70 & $2.61 \mathrm{E}-04$ & $7.21 \mathrm{E}-03$ & & & \\
\hline gga-mir-499 & -1.43 & $9.45 \mathrm{E}-06$ & $4.30 \mathrm{E}-04$ & & & \\
\hline gga-mir-6586 & 3.53 & $1.74 \mathrm{E}-04$ & 5.17E-03 & 3.29 & $3.42 \mathrm{E}-04$ & 1.07E-02 \\
\hline gga-mir-9-1 & -1.24 & 3.97E-09 & $3.41 \mathrm{E}-07$ & & & \\
\hline gga-mir-9-1* & -1.24 & $3.97 \mathrm{E}-09$ & $3.41 \mathrm{E}-07$ & & & \\
\hline gga-mir-9-2 & -1.24 & 3.97E-09 & $3.41 \mathrm{E}-07$ & & & \\
\hline novelMiR_102 & 3.83 & $2.23 \mathrm{E}-05$ & $9.09 \mathrm{E}-04$ & 3.64 & $3.46 \mathrm{E}-05$ & $1.69 \mathrm{E}-03$ \\
\hline novelMiR_117 & -1.34 & $6.69 \mathrm{E}-05$ & $2.16 \mathrm{E}-03$ & & & \\
\hline novelMiR_1232 & -3.24 & $1.12 \mathrm{E}-03$ & $2.48 \mathrm{E}-02$ & 3.67 & $3.39 \mathrm{E}-05$ & $1.69 \mathrm{E}-03$ \\
\hline novelMiR_1241 & -4.02 & $6.36 \mathrm{E}-06$ & $3.28 \mathrm{E}-04$ & & & \\
\hline novelMiR_1251 & -11.45 & $8.82 \mathrm{E}-08$ & $5.69 \mathrm{E}-06$ & 11.29 & $5.67 E-101$ & $4.43 \mathrm{E}-98$ \\
\hline novelMiR_1279_1 & -5.26 & $2.57 \mathrm{E}-05$ & $9.47 \mathrm{E}-04$ & 4.03 & $2.95 \mathrm{E}-07$ & 1.77E-05 \\
\hline novelMiR_1279_2 & -5.26 & $2.57 \mathrm{E}-05$ & $9.47 \mathrm{E}-04$ & 4.03 & $2.95 \mathrm{E}-07$ & 1.77E-05 \\
\hline novelMiR_129_1 & -1.24 & 3.97E-09 & $3.41 \mathrm{E}-07$ & & & \\
\hline novelMiR_129_2 & -1.24 & $3.97 \mathrm{E}-09$ & $3.41 \mathrm{E}-07$ & & & \\
\hline novelMiR_130 & 3.49 & $2.26 \mathrm{E}-04$ & $6.48 \mathrm{E}-03$ & & & \\
\hline novelMiR_1512_1 & -4.46 & 3.97E-09 & $3.41 \mathrm{E}-07$ & & & \\
\hline novelMiR_1565 & -5.95 & $4.94 \mathrm{E}-24$ & $1.91 \mathrm{E}-21$ & -6.35 & $4.31 \mathrm{E}-21$ & $1.12 \mathrm{E}-18$ \\
\hline novelMiR_1616 & -2.96 & $1.02 \mathrm{E}-03$ & $2.32 \mathrm{E}-02$ & & & \\
\hline novelMiR_203_4 & -8.05 & $3.49 \mathrm{E}-04$ & $9.31 \mathrm{E}-03$ & & & \\
\hline novelMiR_277 & -2.81 & $1.89 \mathrm{E}-03$ & 4.06E-02 & & & \\
\hline novelMiR_329 & 3.96 & $4.81 \mathrm{E}-04$ & $1.16 \mathrm{E}-02$ & & & \\
\hline novelMiR_369 & -3.54 & $3.28 \mathrm{E}-05$ & $1.10 \mathrm{E}-03$ & & & \\
\hline novelMiR_396 & -7.10 & $6.58 \mathrm{E}-47$ & $5.09 \mathrm{E}-44$ & & & \\
\hline novelMiR_434 & 4.13 & $3.85 \mathrm{E}-04$ & $9.93 \mathrm{E}-03$ & & & \\
\hline novelMiR_443 & 3.88 & $5.57 \mathrm{E}-04$ & $1.31 \mathrm{E}-02$ & & & \\
\hline novelMiR_451 & -3.61 & 1.87E-05 & $8.05 E-04$ & & & \\
\hline novelMiR_459 & -4.81 & 4.00E-04 & $9.99 \mathrm{E}-03$ & 3.62 & $1.98 \mathrm{E}-04$ & $6.72 \mathrm{E}-03$ \\
\hline novelMiR_464 & -4.14 & $2.78 \mathrm{E}-05$ & $9.77 \mathrm{E}-04$ & -4.17 & $3.29 E-06$ & $1.83 \mathrm{E}-04$ \\
\hline novelMiR_483 & 4.10 & $2.31 \mathrm{E}-06$ & 1.37E-04 & & & \\
\hline novelMiR_488 & -7.15 & $3.70 \mathrm{E}-08$ & $2.60 \mathrm{E}-06$ & 4.02 & $6.46 \mathrm{E}-10$ & $6.31 \mathrm{E}-08$ \\
\hline novelMiR_909 & 7.64 & $3.66 \mathrm{E}-06$ & $2.03 E-04$ & & & \\
\hline gga-mir-1434 & & & & 2.42 & $1.25 \mathrm{E}-03$ & $3.29 \mathrm{E}-02$ \\
\hline gga-mir-1655 & & & & 2.94 & 1.97E-03 & 4.80E-02 \\
\hline gga-mir-1798 & & & & 3.10 & $1.02 \mathrm{E}-03$ & $2.84 \mathrm{E}-02$ \\
\hline novelMiR_145_1 & & & & 2.86 & 7.47E-14 & $9.72 \mathrm{E}-12$ \\
\hline novelMiR_145_2 & & & & 2.86 & $7.33 \mathrm{E}-14$ & $9.72 \mathrm{E}-12$ \\
\hline novelMiR_215 & & & & -5.71 & $4.45 \mathrm{E}-15$ & $8.68 \mathrm{E}-13$ \\
\hline novelMiR_268 & & & & 2.41 & $5.20 \mathrm{E}-04$ & $1.51 \mathrm{E}-02$ \\
\hline novelMiR_474 & & & & 11.06 & $2.34 \mathrm{E}-08$ & $1.66 \mathrm{E}-06$ \\
\hline novelMiR_475 & & & & 8.01 & $1.29 \mathrm{E}-04$ & $5.05 E-03$ \\
\hline novelMiR_5 & & & & 2.85 & 1.17E-13 & $1.31 \mathrm{E}-11$ \\
\hline novelMiR_507_1 & & & & 3.46 & $1.04 \mathrm{E}-04$ & 4.27E-03 \\
\hline novelMiR_507_2 & & & & 3.42 & $1.39 E-04$ & $5.16 \mathrm{E}-03$ \\
\hline
\end{tabular}


Table 3 (continued)

\begin{tabular}{|c|c|c|c|c|c|c|}
\hline \multirow[t]{3}{*}{ miRNA_ID } & \multicolumn{6}{|c|}{ Line_ $6 \sigma_{3} /$ Line $\_72$} \\
\hline & \multicolumn{3}{|c|}{ HVT-induced } & \multicolumn{3}{|c|}{ Rispens-induced } \\
\hline & $\log _{2} F C$ & $p$ value & $F D R$ & $\log _{2} F C$ & $p$ value & $F D R$ \\
\hline novelMiR_507_3 & & & & 3.46 & $1.04 \mathrm{E}-04$ & 4.27E-03 \\
\hline novelMiR_507_4 & & & & 3.46 & $1.04 \mathrm{E}-04$ & 4.27E-03 \\
\hline novelMiR_52 & & & & 2.80 & $3.01 \mathrm{E}-09$ & $2.35 \mathrm{E}-07$ \\
\hline novelMiR_60 & & & & -3.19 & $1.59 \mathrm{E}-03$ & $4.01 \mathrm{E}-02$ \\
\hline novelMiR_97_1 & & & & 1.45 & $2.64 \mathrm{E}-04$ & $8.58 \mathrm{E}-03$ \\
\hline novelMiR_97_2 & & & & 9.08 & $1.97 \mathrm{E}-66$ & $7.69 E-64$ \\
\hline
\end{tabular}

Table 4 Primers used in ddPCR to validate the expressions of a small subgroup of identified miRNAs, which were determined by small RNA_Seq analysis

\begin{tabular}{|c|c|c|c|}
\hline miRNA & Sequence & Forward primer $\left(5^{\prime} \rightarrow 3^{\prime}\right)$ & Reverse primer $\left(5^{\prime} \rightarrow 3^{\prime}\right)$ \\
\hline gga-miR-31 & aggcaagatgttggcatagctg & gcagaggcaagatgttggcat & caggtccagttttttttttttttcagcta \\
\hline gga-miR-193b & aactggeccacaaagtcccgct & cgcagaactggcccacaaag & gtccagttttttttttttttagcgggact \\
\hline gga-mir-140 & accacagggtagaaccacggac & cagaccacagggtagaacca & aggtccagttttttttttttttgtccgt \\
\hline gga-mir-142 & cataaagtagaaagcactact & cagcgcagcataaagtagaaagca & gcaggtccagtttttttttttttttagtagt \\
\hline gga-miR-499 & ttaagacttgtagtgatgttt & agcgcagttaagacttgtagtgat & agcaggtccagtttttttttttttttaaacat \\
\hline gga-mir-153 & ttgcatagtcacaaaagtgatc & gcgcagttgcatagtcacaaaag & ccaggtccagttttttttttttttgatca \\
\hline gga-mir-1677 & ttgacttcagtaggagcagggatt & gcagttgacttcagtaggagca & gcaggtccagtttttttttttttttaatcct \\
\hline gga-mir-1769 & agtgtgaaatctgcctgaaagt & gcagagtgtgaaatctgcctga & gcaggtccagtttttttttttttttactttc \\
\hline novelMiR_142 & agccggggatgattitctgcct & cgcagagccggggatgatt & aggtccagttttttttttttttaggcagaa \\
\hline novelMiR-5 & gtagtcgtggccgagtggttaag & ggtagtcgtggecgagtg & gcaggtccagtttttttttttttttcttaac \\
\hline
\end{tabular}

Table 5 Summary statistics of ddPCR absolute counts and RNA_Seq reads for validation of miRNA expression data detected by small RNA sequencing

\begin{tabular}{llll}
\hline miRNA & No. observations & $\boldsymbol{r}$ & $\boldsymbol{p}$ value \\
\hline gga-miR-31 & 24 & 0.99 & 0.0001 \\
gga-miR-193b & 24 & 0.94 & 0.0001 \\
gg-miR-140 & 24 & 0.63 & 0.001 \\
gga-miR-142 & 24 & 0.87 & 0.0001 \\
gga-miR-499 & 24 & 0.99 & 0.0001 \\
gga-miR-153 & 24 & 0.85 & 0.0001 \\
gga-miR-1677 & 24 & 0.77 & 0.0001 \\
gga-miR-1769 & 24 & 0.98 & 0.0001 \\
novelMiR-142 & 24 & 0.79 & 0.0001 \\
nvelMiR-5 & 24 & 0.98 & 0.0001 \\
\hline
\end{tabular}

receptor signaling pathway, ErbB signaling pathway, FoxO signaling pathway, mRNA surveillance pathway, mTOR signaling pathway, and TFG-beta signaling pathway (Additional file 8). Additional GO terms and pathways for the target genes from comparisons between CVI988/Rispens and HVT in lines $6_{3}$ and $7_{2}$, between lines $6_{3}$ and $7_{2}$ in response to CVI988/Rispens, and line
$7_{2}$ birds in response to CVI988/Rispens are given in rest of the Additional files 9, 10, 11, 12 .

\section{Discussion}

Marek's disease has been well under controlled since the 1970s, which, in large, is attributable to the wide use of MD vaccines in poultry flocks [9]. Commonly used commercial $\mathrm{MD}$ vaccines include the first-generation anti-virus-induced tumor vaccine HVT, then the second comer SB-1, and the current gold-standard MD vaccine CVI988/Rispens [39, 40]. While most researchers and industry professionals, if not all, fully recognize the great good that $\mathrm{MD}$ vaccines have done for the poultry industry, few, if any, claim how the MD vaccines protect chickens against the MDV-induced tumors is thoroughly understood, including immunologists [41]. The reality that this paradox persistently remains bars the advancement of knowledge-based new vaccine design and development.

Genetic mechanism underlying how MD vaccine inserts protection against MD tumor formation is also far from fully understood. Earlier studies demonstrated that $M H C$ plays an important role in modulating MD vaccine protective efficacy in chicken [21-23, 42, 43]. Our recent 

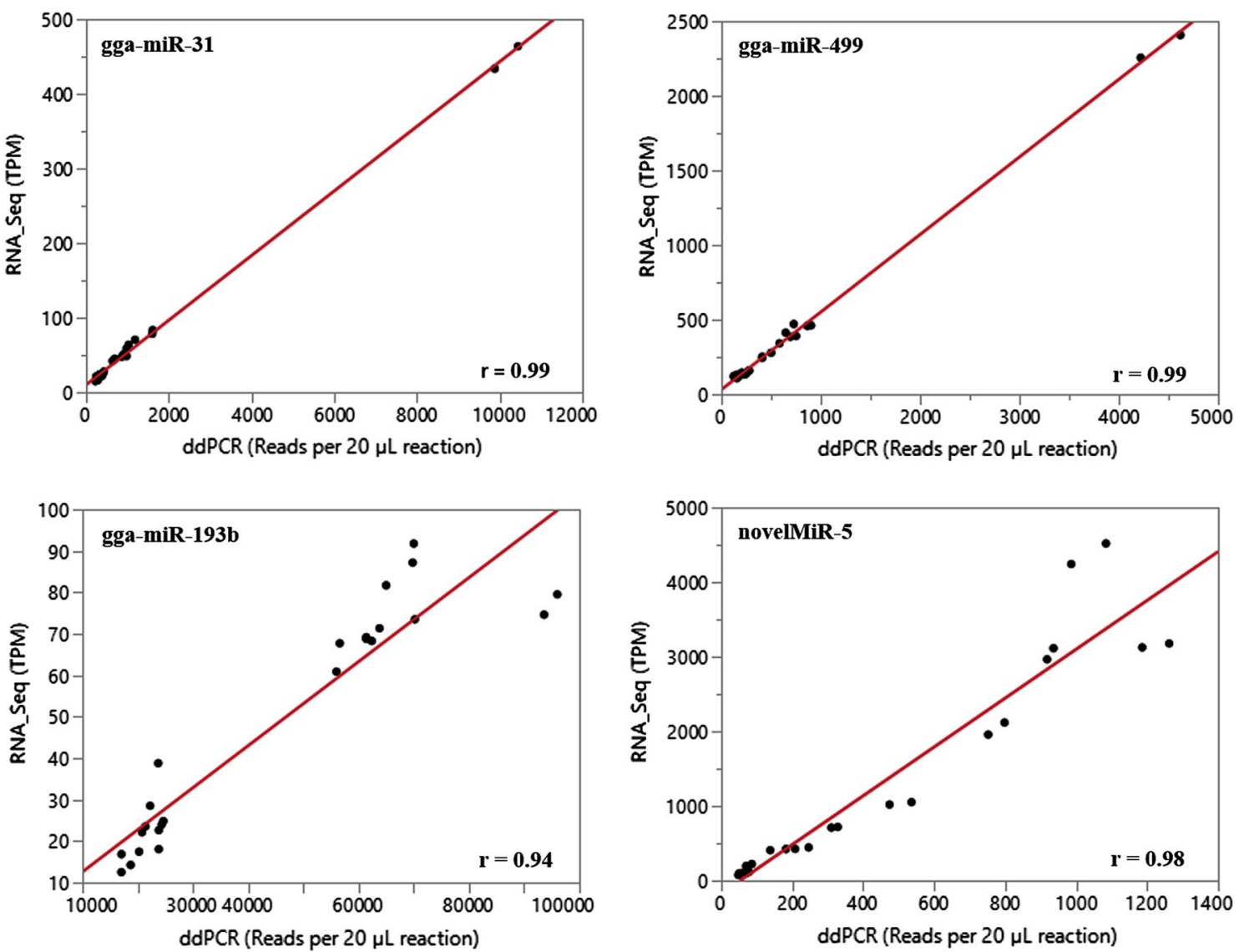

Figure 3 Bivariate plots illustrating the relationship between small RNA_Seq data and droplet digital PCR data. The normalized expression of small RNA_Seq data, transcripts per million, (TPM), and droplet digital PCR (ddPCR) data were analyzed by fitting a bivariate model. The bivariate plots for four of the validated miRNAs are given graphically showing the two sets of expression data. The correlation coefficients between the small RNA_Seq and the ddPCR data for these four miRNAs ranged from $r=0.94$ (gga-miR-193b) to $r=0.99$ (both gga-miR-31 and gga-miR-499), which provided highly positive support to the estimates of microRNA expression derived from the small RNA_Seq data.

studies using the highly inbred lines of chickens, lines $6_{3}$ and $7_{2}$ carrying the same $M H C B^{*} 2$ haplotype, showed that non- $M H C$ genomic contents also play a significant role in enabling chicken's ability to convey protection against MDV-induced tumor formation in response to $M D$ vaccines. Our experimental data clearly showed the line $6_{3}$ birds convey a very high protection rate, which strikingly differs from the line $7_{2}$ birds in response to HVT. The CVI988/Rispens delivers similar protection to the line $6_{3}$ but a little better protection to line $7_{2}$ than HVT $[25,28]$.

Epigenetics has been demonstrated to play an important role in orchestrating key biological processes, which are implemented through a number of factors including DNA methylation, histone modification, and ncRNAs $[44,45]$. The epigenetic factors, in turn, are continuously modified throughout life in response to environmental exposures [45, 46]. Epigenetics refers to the study of changes in gene expression that occur without a change in DNA sequence. DNA methylation involves the addition of a methyl group to the carbon- 5 of the cytosine pyrimidine ring and typically occurs at $\mathrm{CpG}$ sites containing cytosine-guanine nucleotides in a linear sequence. $\mathrm{CpG}$ islands, short stretches of DNA with rich CpG sites, are often found at promoters of mammalian genes. DNA methylation at these sites is highly correlated with transcription status of corresponding genes. Histone modification defines discrete chromatin regions with distinct structures associated with distinct transcription states of genes. miRNAs are small-non-coding RNAs. Mature miRNAs are 23 nucleotides in length and regulate gene expression by preventing the translation of specific mRNAs. Epigenetic mechanisms are multifaceted and complex, which provide an additional layer of transcriptional control and a layer of post-transcriptional control to regulate gene expression, and, consequently, gene function [47-52]. A very recent study using small RNA_Seq identified 24 cellular miRNAs with altered 


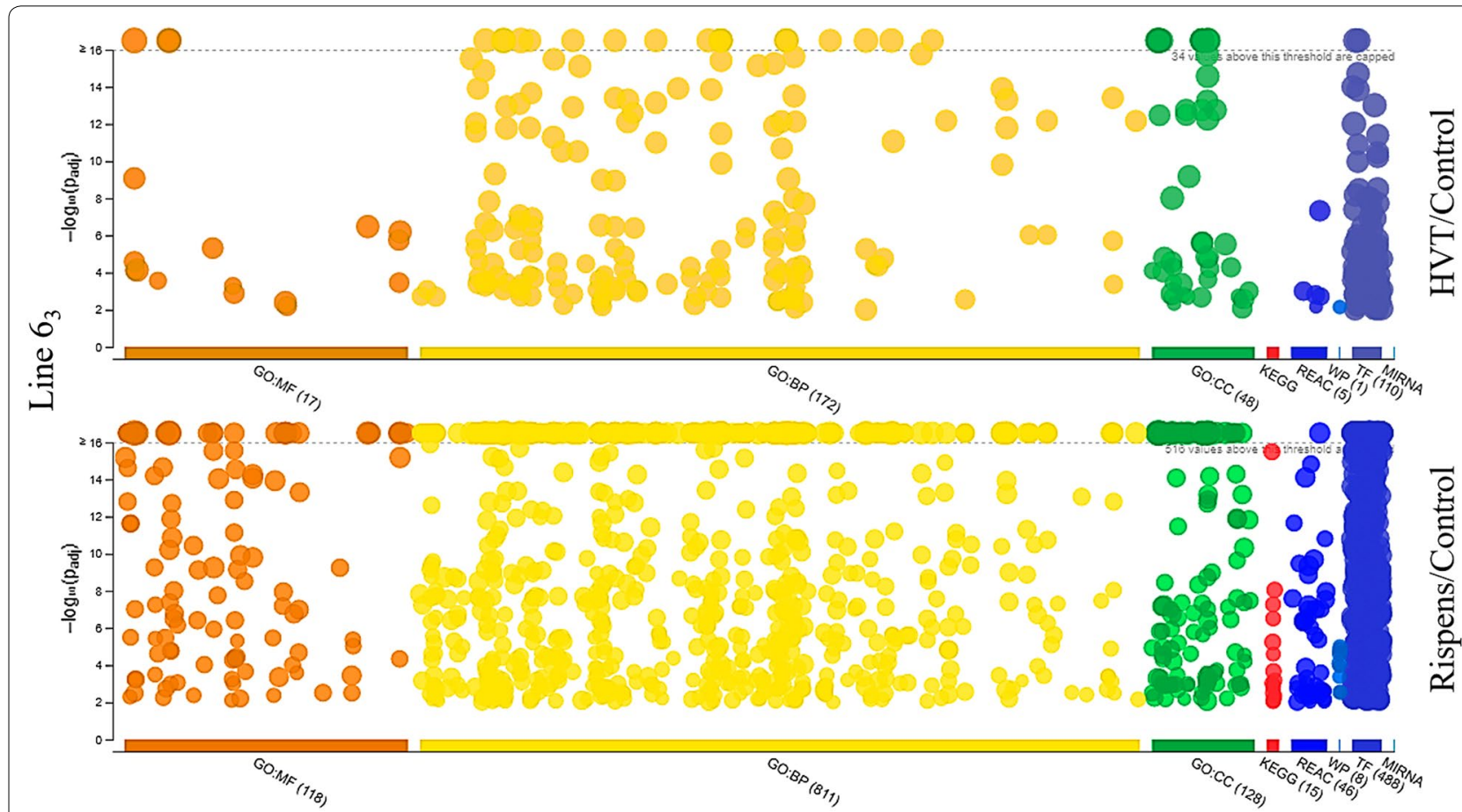

Figure 4 Manhattan plots illustrating GO term enrichments of target genes. The target genes of differentially expressed microRNAs of the line $\sigma_{3}$ birds were analyzed by g:Profiler and the enrichment in GO terms (MF: molecular function; BP: biological process; CC: cellular component) and KEGG pathways across Reactome pathways (REAC), WiKi-Pathways (WP), transcription factor (TF), and microRNA target base (MIRNA) were graphically depicted in two plots. The plots depicted the enrichments of target genes for differentially expressed microRNAs of the line $\sigma_{3}$ birds in response to HVT (top) and CVI988/Rispens (bottom) vaccination, respectively. A clearly visible difference in enrichments between the two treatment groups (HVT and CVI988/Rispens vaccination) is also demonstrated.

Table 6 Identified numbers of differentially expressed miRNAs, predicted target genes, enriched in number of GO terms and KEGG pathways per contrast group

\begin{tabular}{|c|c|c|c|c|}
\hline Contrast & $\begin{array}{l}\text { Differentially expressed } \\
\text { miRNAs }\end{array}$ & Predicted target genes & Number of GO terms & $\begin{array}{l}\text { Number of KEGG } \\
\text { pathways }\end{array}$ \\
\hline Line $\sigma_{3} H V T /$ control & 4 & 463 & 237 & 0 \\
\hline Line $\sigma_{3}$ rispens/control & 13 & 2928 & 1057 & 15 \\
\hline Line $7_{2} \mathrm{HVT} /$ control & 0 & 0 & 0 & 0 \\
\hline Line $7_{2}$ rispens/control & 1 & 12 & 4 & 1 \\
\hline Line $\sigma_{3}$ rispens/HVT & 28 & 3677 & 1255 & 24 \\
\hline Line $7_{2}$ rispens/HVT & 2 & 176 & 113 & 0 \\
\hline Line $\sigma_{3} \mathrm{HVT} /$ Line $7_{2} \mathrm{HVT}$ & 34 & 6153 & 1999 & 46 \\
\hline Line $\sigma_{3}$ rispens/line $7_{2}$ rispens & 30 & 5277 & 1577 & 32 \\
\hline
\end{tabular}

expression in response to HVT, MDV, or both inoculations. The report claims cellular miRNAs are of critical players both in protection against and mediating progression of MD [53].

Up to date, there are 2114 unique gga-miRNAs that have been coined with accession numbers (miRbase release 22.1: October 2018). This study identified a total of 693 miRNAs including 198 reported gga-miRNAs and 495 novel miRNAs in the line $6_{3}$ and $7_{2}$ chickens.
The identified novelMiRs were over twice as many as the identified known gga-miRNAs in bursae of the line $6_{3}$ and $7_{2}$ chickens 26 days post MD vaccine inoculation (Additional file 1).

Notable difference in the number of differentially expressed miRNAs were observed in the line $6_{3}$ birds. Vaccine and challenge trials repeatedly showed that HVT provides equally well or even better protection against MD in response to very virulent plus MDV 
challenge [25, 28], yet only four differentially expressed miRNAs were identified in line $6_{3}$ in response to HVT inoculation, but 13 differentially expressed miRNAs were identified in response to CVI988/Rispens in this line. Further, a direct comparison between CVI988/ Rispens and HVT inoculation of line $6_{3}$ birds resulted in a total of 28 differentially expressed miRNAs. All these differentially expressed miRNAs (Table 1) might in different ways at different levels through their target genes as well as the GO terms and pathways insert influence to mediate each vaccine's protective efficacy against MD.

In contrast, direct comparisons between the lines $6_{3}$ and $7_{2}$ birds 26 days post-vaccination resulted in a total of 34 and 30 differentially expressed miRNAs in response to HVT and CVI988/Rispens inoculation, respectively (Table 3 ). It is interesting to note that the four differentially expressed miRNAs identified between line $6_{3} \mathrm{HVT}$ and line $6_{3}$ control groups (Table 1) were also among the 34 differentially expressed miRNAs of the line $6_{3}$ and $7_{2}$ comparison inoculated with HVT. Not only so, the $\log _{2}$ FC positive and negative signs of the four differentially miRNAs were also consistent, and the magnitude of fold change were similar. Knowing that HVT does not induce much protection to line $7_{2}$ but great protection for line $6_{3}$ birds, it is speculated here that the additional 30 differentially expressed miRNAs identified between lines $6_{3}$ and $7_{2}$ post HVT inoculation might be also important in modulating HVT protective efficacy in chickens like the line $6_{3}$ birds.

In summary, a relatively large number of differentially expressed microRNAs were identified in two highly inbred lines of chicken post MD vaccine inoculation. These two genetic lines of chickens differ in the capacity to convey protective efficacy against MDV-induced tumor formation in response to MD vaccination based on previous studies. Therefore, this finding may serve as the first piece of experimental evidence that the epigenetic factor, microRNA, is highly likely involved in modulating vaccine protective efficacy in chicken through their target genes in combination with varied biological processes and pathways.

\section{Supplementary information}

Supplementary information accompanies this paper at https://doi. org/10.1186/s13567-020-00746-4.

Additional file 1: Complete list of unique microRNAs identified in al biosamples of this study. Unique microRNAs including known microRNAs and novel microRNAs identified in two lines of chickens in response to vaccination
Additional file 2: miRNAs identified in bursae of Line $\sigma_{3}$ chickens 26 days post HVT inoculation. A microRNA profile of line $\sigma_{3}$ in response to HVT vaccination.

Additional file 3: miRNAs identified in bursae of Line $\sigma_{3}$ chickens 26 days post Rispens inoculation. A microRNA profile of line $\sigma_{3}$ in response to CVI988/Rispens vaccination.

Additional file 4: miRNAs identified in bursae of Line $\mathbf{7}_{\mathbf{2}}$ chickens 26 days post HVT inoculation. A microRNA profile of line $7_{2}$ in response to HVT vaccination.

Additional file 5: miRNAs identified in bursae of Line $\mathbf{7}_{2}$ chickens 26 days post Rispens inoculation. A microRNA profile of line $7_{2}$ in response to CVI988/Rispens vaccination.

Additional file 6: GO terms enriched by target genes of differentially expressed miRNAs in line $\sigma_{3}$ bursae 26 days post HVT inoculation. Gene Ontology analysis of a list of target genes of differentially expressed microRNAs of the line $\sigma_{3}$ birds in response to HVT vaccination.

Additional file 7: GO terms enriched by target genes of differentially expressed miRNAs in line $6_{3}$ bursae 26 days post Rispens inoculation. Gene Ontology analysis of a list of target genes of differentially expressed microRNAs of the line $\sigma_{3}$ birds in response to CVI988/Rispens vaccination.

Additional file 8: GO terms enriched by target genes of differentially expressed miRNAs in bursae between line $6_{3}$ and line $7_{2}$ birds 26 days post HVT inoculation. Gene Ontology analysis of a list of target genes of differentially expressed microRNAs between line $\sigma_{3}$ and line $7_{2}$ birds 26 days post HVT vaccination.

Additional file 9: GO terms enriched by target genes of differentially expressed miRNAs in bursae between Rispens and HVT vaccinated line $\mathbf{6}_{\mathbf{3}}$ birds $\mathbf{2} 6$ days post-inoculation. Gene Ontology analysis of a list of target genes of differentially expressed microRNAs between Rispens and HVT inoculated line $\sigma_{3}$ birds 26 days post-vaccination.

Additional file 10: GO terms enriched by target genes of differentially expressed miRNAs in bursae between Rispens and HVT vaccinated line $\mathbf{7}_{2}$ birds $\mathbf{2 6}$ days post-inoculation. Gene Ontology analysis of a list of target genes of differentially expressed microRNAs between Rispens and HVT vaccinated line $7_{2}$ birds 26 days post-vaccination.

Additional file 11: GO terms enriched by target genes of differentially expressed miRNAs in bursae between line $6_{3}$ and line $7_{2}$ birds 26 days post Rispens inoculation. Gene Ontology analysis of a list of target genes of differentially expressed microRNAs between line $\sigma_{3}$ and line 72 birds 26 days post CVI988/Rispens vaccination.

Additional file 12: GO terms enriched by target genes of a differentially expressed miRNA in bursae of line $7_{2}$ birds in response to Rispens vaccination. Gene Ontology analysis of a list of target genes of a differentially expressed microRNA of line 72 birds 26 days post CVI988/ Rispens vaccination.

\section{Acknowledgements}

Dr Jiyuan An is gratefully appreciated and acknowledged for the construction of chicken genome build index files used in miRDeep* analysis of the chicken small RNA_Seq data. The authors also thank Bernice Li and Yanfen Zhai for their excellent technical support on conducting the animal experiment, tissue sampling, ddPCR analysis, and GO term enrichment work; and Melanie Flesberg, on total RNA extraction, qualification, and quantification.

\section{Authors' contributions}

LZ and CZ conducted bioinformatic analyses of the small RNA sequence dataset, prepared the draft of the manuscript, tabulated and depicted the results. $\mathrm{MH}$ revised the manuscript. $\mathrm{KD}$ prepared the $\mathrm{R}$ customer scripts and revised the manuscript. SC and QX participated in the animal experimental design, helped conduct the animal experiment, and revised the manuscript. $\mathrm{HZ}$ conceived and supervised this study. All authors read and approved the final manuscript. 


\section{Funding}

This work was solely supported by Congressional Prepared Budget for Research ministered by US department of Agriculture and Agricultural Research Service. Lei Zhang was financially supported by the China Scholarship Council (No. 201803250035) sponsoring visiting scholars studying abroad. Mention of trade names or commercial products in this publication is solely for the purpose of providing specific information and does not imply recommendation or endorsement by the U.S. Department of Agriculture

\section{Competing interests}

The authors declare that they have no competing interests.

\section{Author details}

${ }^{1}$ Avian Disease and Oncology Laboratory, USDA-ARS, East Lansing, MI 48823 USA. ${ }^{2}$ Institute of Special Animal and Plant Sciences, Chinese Academy of Agricultural Sciences, Changchun, Jilin 130112, China. ${ }^{3}$ Michigan State University, East Lansing, MI 48824, USA. ${ }^{4}$ Department of Pharmacology and Toxicology, Augusta University, Augusta, GA 30912, USA. ${ }^{5}$ College of Veterinary Medicine, Shandong Agricultural University, Tai'an, Shandong 271018 China. ${ }^{6}$ College of Animal Science, South China Agricultural University, Guangzhou 510642, China.

Received: 25 September 2019 Accepted: 27 January 2020

Published online: 24 February 2020

\section{References}

1. Marek J (1907) Multiple Nervenentzündung (Polyneuritis) bei Hühnern. Dtsch Tierärztl Wochenschr 15:417-421

2. Gimeno IM, Witter RL, Reed WM (1999) Four distinct neurologic syndromes in Marek's disease: effect of viral strain and pathotype. Avian Dis 43:721-737

3. Xie QM, Chang S, Dong KZ, Dunn JR, Song JZ, Zhang HM (2017) Genomic variation between genetic lines of White Leghorns differed in resistance to Marek's disease. J Clin Epigenet 3:7

4. Niu S, Jahejo AR, Jia FJ, Li X, Ning GB, Zhang D, Ma HL, Hao WF, Gao WW Zhao YJ, Gao SM, Li GL, Li JH, Yan F, Gao RK, Bi YH, Han LX, Gao GF, Tian WX (2018) Transcripts of antibacterial peptides in chicken erythrocytes infected with Marek's disease virus. BMC Vet Res 14:363

5. Calnek BW (2001) Pathogenesis of Marek's disease virus infection. Curr Top Microbiol Immunol 255:25-55

6. Brochu NM, Guerin MT, Varga C, Lillie BN, Brash ML, Susta L (2019) A twoyear prospective study of small poultry flocks in Ontario, Canada, part 2: causes of morbidity and mortality. J Vet Diagn Invest 31:336-342

7. Gimeno IM (2008) Marek's disease vaccines: a solution for today but a worry for tomorrow? Vaccine 26(Suppl 3):C31-41

8. Rozins C, Day T, Greenhalgh S (2019) Managing Marek's disease in the egg industry. Epidemics 27:52-58

9. Biggs PM, Nair V (2012) The long view: 40 years of Marek's disease research and Avian Pathology. Avian Pathol 41:3-9

10. Smith J, Sadeyen JR, Paton IR, Hocking PM, Salmon N, Fife M, Nair V, Burt DW, Kaiser $P$ (2011) Systems analysis of immune responses in Marek's disease virus-infected chickens identifies a gene involved in susceptibility and highlights a possible novel pathogenicity mechanism. J Virol 85:11146-11158

11. Schat KA, Calnek BW (1978) Protection against Marek's disease-derived tumor transplants by the nononcogenic SB-1 strain of Marek's disease virus. Infect Immun 22:225-232

12. Rispens $B H$, van Volten $H$, Mastenbroek N, Maas JL, Schat KA (1972) Control of Marek's disease in the Netherlands. II. Field trials on vaccination with an avirulent strain (CVI 988) of Marek's disease virus. Avian Dis $16: 126-138$

13. Witter RL, Offenbecker $L$ (1978) Duration of vaccinal immunity against Marek's disease. Avian Dis 22:396-408

14. Baigent SJ, Smith LP, Nair VK, Currie RJ (2006) Vaccinal control of Marek's disease: current challenges, and future strategies to maximize protection. Vet Immunol Immunopathol 112:78-86

15. Wozniakowski G, Niczyporuk JS (2015) Detection of specific UL49 sequences of Marek's disease virus CVI988/Rispens strain using loopmediated isothermal amplification. J Virol Methods 221:22-28
16. Baigent SJ, Nair VK, Le Galludec H (2016) Real-time PCR for differential quantification of CVI988 vaccine virus and virulent strains of Marek's disease virus. J Virol Methods 233:23-36

17. Liu S, Sun W, Huang X, Zhang W, Jia C, Luo J, Shen Y, El-Ashram S, He $C$ (2017) A promising recombinant herpesvirus of turkeys vaccine expressing PmpD-N of Chlamydia psittaci based on elongation factor-1 alpha promoter. Front Vet Sci 4:221

18. McKimm-Breschkin JL, Faragher JT, Withell J, Forsyth WM (1990) Isolation of very virulent Marek's disease viruses from vaccinated chickens in Australia. Aust Vet J 67:205-209

19. Sharma JM, Witter RL (1983) Embryo vaccination against Marek's disease with serotypes 1,2 and 3 vaccines administered singly or in combination. Avian Dis 27:453-463

20. Spencer JL, Robertson A (1975) Influence of vaccination with avirulent herpesvirus on subsequent infection of chickens with virulent Marek's disease herpesvirus. Am J Vet Res 36:1235-1239

21. Bacon LD, Hunt HD, Cheng HH (2000) A review of the development of chicken lines to resolve genes determining resistance to diseases. Poult Sci 79:1082-1093

22. Bacon LD, Witter RL (1994) Serotype specificity of B-haplotype influence on the relative efficacy of Marek's disease vaccines. Avian Dis 38:65-71

23. Bacon LD, Witter RL (1994) B haplotype influence on the relative efficacy of Marek's disease vaccines in commercial chickens. Poult Sci 73:481-487

24. Witter RL (2001) Protective efficacy of Marek's disease vaccines. Curr Top Microbiol Immunol 255:57-90

25. Chang S, Xie Q, Dunn JR, Ernst CW, Song J, Zhang HM (2014) Host genetic resistance to Marek's disease sustains protective efficacy of herpesvirus of turkey in both experimental and commercial lines of chickens. Vaccine 32:1820-1827

26. Tomasi TB, Magner WJ, Khan AN (2006) Epigenetic regulation of immune escape genes in cancer. Cancer Immunol Immunother 55:1159-1184

27. Dougan G, Highfield P (1985) Molecular biology, the new genetics and vaccine development. Med Lab Sci 42:393-398

28. Chang S, Dunn JR, Heidari M, Lee LF, Song J, Ernst CW, Ding Z, Bacon LD, Zhang H (2010) Genetics and vaccine efficacy: host genetic variation affecting Marek's disease vaccine efficacy in White Leghorn chickens. Poult Sci 89:2083-2091

29. Heidari M, Zhang HM (2019) PRJNA543524. NCBI. https://www.ncbi.nlm. nih.gov/sra

30. An J, Lai J, Lehman ML, Nelson CC (2013) miRDeep*: an integrated application tool for miRNA identification from RNA sequencing data. Nucleic Acids Res 41:727-737

31. Kozomara A, Birgaoanu M, Griffiths-Jones S (2019) miRBase: from microRNA sequences to function. Nucleic Acids Res 47:D155-D162

32. Kozomara A (2019) miRBase. http://www.mirbase.org/ftp.shtm

33. Lewis BP, Burge CB, Bartel DP (2005) Conserved seed pairing, often flanked by adenosines, indicates that thousands of human genes are microRNA targets. Cell 120:15-20

34. Balcells I, Cirera S, Busk PK (2011) Specific and sensitive quantitative RTPCR of miRNAs with DNA primers. BMC Biotechnol 11:70

35. Anders S, Pyl PT, Huber W (2015) HTSeq-a Python framework to work with high-throughput sequencing data. Bioinformatics 31:166-169

36. g:Profiler (2019) ELIXIR. http://biit.cs.ut.ee/gprofiler/index.cgi

37. Reimand J, Kull M, Peterson H, Hansen J, Vilo J (2007) g:Profiler-a webbased toolset for functional profiling of gene lists from large-scale experiments. Nucleic Acids Res 35:W193-200

38. Zhang HM, Heidari M (2019) PRJNA543526. NCBI. https://www.ncbi.nlm. nih.gov/sra/PRJNA543526

39. Garcia M (2017) Current and future vaccines and vaccination strategies against infectious laryngotracheitis (ILT) respiratory disease of poultry. Vet Microbiol 206:157-162

40. Reddy SM, Izumiya Y, Lupiani B (2017) Marek's disease vaccines: current status, and strategies for improvement and development of vector vaccines. Vet Microbiol 206:113-120

41. Boodhoo N, Gurung A, Sharif S, Behboudi S (2016) Marek's disease in chickens: a review with focus on immunology. Vet Res 47:119

42. Bacon LD, Witter RL (1992) Influence of turkey herpesvirus vaccination on the B-haplotype effect on Marek's disease resistance in 15.B-congenic chickens. Avian Dis 36:378-385 
43. Bacon LD, Witter RL (1993) Influence of B-haplotype on the relative efficacy of Marek's disease vaccines of different serotypes. Avian Dis 37:53-59

44. Aslani S, Jafari N, Javan MR, Karami J, Ahmadi M, Jafarnejad M (2017) Epigenetic modifications and therapy in multiple sclerosis. Neuromol Med 19:11-23

45. van Otterdijk SD, Michels KB (2016) Transgenerational epigenetic inheritance in mammals: how good is the evidence? FASEB J 30:2457-2465

46. Marsit CJ (2015) Influence of environmental exposure on human epigenetic regulation. J Exp Biol 218:71-79

47. Hamilton JP (2011) Epigenetics: principles and practice. Dig Dis 29:130-135

48. Ling C, Groop L (2009) Epigenetics: a molecular link between environmental factors and type 2 diabetes. Diabetes 58:2718-2725

49. Reddy MA, Natarajan R (2011) Epigenetic mechanisms in diabetic vascular complications. Cardiovasc Res 90:421-429
50. van Weerd JH, Koshiba-Takeuchi K, Kwon C, Takeuchi JK (2011) Epigenetic factors and cardiac development. Cardiovasc Res 91:203-211

51. Wulff BE, Nishikura K (2012) Modulation of microRNA expression and function by ADARs. Curr Top Microbiol Immunol 353:91-109

52. Yan C, Boyd DD (2006) Histone H3 acetylation and H3 K4 methylation define distinct chromatin regions permissive for transgene expression. Mol Cell Biol 26:6357-6371

53. Hicks JA, Liu HC (2019) Impact of HVT vaccination on splenic miRNA expression in Marek's disease virus infections. Genes (Basel) 10:E115

\section{Publisher's Note}

Springer Nature remains neutral with regard to jurisdictional claims in published maps and institutional affiliations.
Ready to submit your research? Choose BMC and benefit from:

- fast, convenient online submission

- thorough peer review by experienced researchers in your field

- rapid publication on acceptance

- support for research data, including large and complex data types

- gold Open Access which fosters wider collaboration and increased citations

- maximum visibility for your research: over $100 \mathrm{M}$ website views per year

At BMC, research is always in progress.

Learn more biomedcentral.com/submissions 